Discrete Comput Geom 38:231-241 (2007)

DOI: $10.1007 / \mathrm{s} 00454-007-1335-6$

\title{
Polytopes of Minimal Volume with Respect to a Shell-Another Characterization of the Octahedron and the Icosahedron*
}

\author{
Károly Böröczky ${ }^{1}$ and Károly Böröczky, Jr. ${ }^{1,2}$ \\ ${ }^{1}$ Department of Geometry, Roland Eötvös University, \\ Pázmány Péter sétány 1/C, Budapest, H-1117, Hungary \\ boroczky@cs.elte.hu \\ ${ }^{2}$ Alfréd Rényi Institute of Mathematics, \\ PO Box 127, Budapest, H-1364, Hungary \\ carlos@renyi.hu
}

Dedicated to the memory of László Fejes Tóth

\begin{abstract}
Given $r>1$, we search for the convex body of minimal volume or of minimal surface area in $\mathbb{E}^{3}$ that contains a unit ball, and the extreme points are of distance at least $r$ from the centre of the unit ball. We show that the optimal bodies are regular octahedron and icosahedron if $r=\sqrt{3}$ or $r=\sqrt{15-6 \sqrt{5}}$, respectively.
\end{abstract}

\section{Notation and Known Results}

We introduce the notation used throughout the paper. For any notions related to convexity in this paper, consult [8]. We write $o$ to denote the origin in the Euclidean space $\mathbb{E}^{n}$, and $\|\cdot\|$ to denote the corresponding Euclidean norm. Given a set $X \subset \mathbb{E}^{n}$, the affine hull and the convex hull of $X$ are denoted by aff $X$ and conv $X$, respectively, moreover the interior of $X$ is denoted by int $X$. For a compact convex $X$, let $\partial X$ denote the "relative boundary" of $X$; namely, the boundary of $X$ with respect to the topology of aff $X$. The unit ball centred at $o$ is denoted by $B^{n}$, and the boundary of $B^{n}$ is denoted by $S^{n-1}$. As usual we call a compact convex set $C$ with non-empty interior a convex body, and write $V(C)$ to denote its volume, and $S(C)$ to denote its surface area. The two-dimensional Hausdorff measure of a measurable subset $C$ of the boundary of some convex body in $\mathbb{E}^{3}$ is called the area $A(C)$ of $C$.

\footnotetext{
* The first author was supported by OTKA Grants T 043556 and 033752 and the second author was supported by OTKA Grants T 042769, 043520 and 049301.
} 
The following statement is the starting point of our investigation:

Theorem 1.1 (Hajós Lemma). Let $r>1$. Among convex polygons, which contain $B^{2}$, and whose vertices are of distance at least $r$ from $o$, the ones with minimal area are inscribed into $r B^{2}$ in a way that all but at most one side touch $B^{2}$. In addition the analogous statement holds for the minimal perimeter.

Theorem 1.1 was proposed and proved by members of the seminar led by Gy. Hajós around 1960. It was inspired by an earlier result of L. Fejes Tóth (see for example [6]); namely, L. Fejes Tóth solved the case of minimal area for $r=2 / \sqrt{3}$ when the optimal polygon is a regular hexagon. Actually his approach yields the similar characterization of any regular polygon.

Developing further some ideas of Rogers and Molnár, the following characterization of regular polytopes in any dimensions appeared in [5]:

Theorem 1.2 (Böröczky). Let $Q$ be a regular polytope in $\mathbb{E}^{n}$ whose circumcentre is o, and let $r_{i}$ denote the distance of an $i$-face of $Q$ from $o$. If $P$ is any polytope such that $o \in \operatorname{int} P$, and the distance of any $i$-face of $P$ from o is at least $r_{i}$ for $i=0, \ldots, n$ then $V(P) \geq V(Q)$. Moreover, equality holds if and only if $P$ is congruent to $Q$, and its circumcentre is $o$.

While our paper is concerned with the Euclidean space, we quickly review what is known in the spherical space $S^{n}$ and in the hyperbolic space $H^{n}$. Theorem 1.1 holds both in $S^{2}$ and in $H^{2}$ according to Molnár [7], and Theorem 1.2 holds both in $S^{3}$ and in $H^{3}$ according to Böröczky and Máthéné Bognár [5]. In higher dimensions one needs some additional restrictions (see [5]).

Recently Theorem 1.2 has been strengthened by Bezdek [1]:

Theorem 1.3 (Bezdek). Let $Q$ be a regular polytope in $\mathbb{E}^{n}$ whose circumcentre is o, and let $r_{i}$ denote the distance of an $i$-face of $Q$ from o. If $P$ is any polytope such that $o \in \operatorname{int} P$, and the distance of any $i$-face of $P$ from $o$ is at least $r_{i}$ for $i=0, \ldots, n$ then $S(P) \geq S(Q)$. Moreover, equality holds if and only if $P$ is congruent to $Q$, and its circumcentre is $o$.

Theorem 1.3 implies Theorem 1.2 because we have $V(Q)=\left(r_{n-1} / n\right) S(Q)$ and $V(P) \geq\left(r_{n-1} / n\right) S(P)$.

\section{The Main Result of the Paper}

Molnár [7] asked the question whether for certain platonic solids in $\mathbb{E}^{3}$, the condition in Theorem 1.2 on the edges is superfluous? In this paper we show that this is really the case for the octahedron and the icosahedron.

We consider a generalization of Theorem 1.1 where the optimal solution a priori may not be a polytope but a more general convex body. We recall that $x$ is an extreme point 
of a convex body $C$ if it does not lie in the relative interior of any segment contained in $C$. Actually the extreme points form the minimal subset of $C$ whose convex hull is $C$.

Definition. Given $r>1$, we write $\mathcal{F}_{r}$ to denote the family of convex bodies in $\mathbb{E}^{3}$, which contain $B^{3}$, and whose extreme points are of distance at least $r$ from $o$. Moreover, let $P_{r} \in \mathcal{F}_{r}$ have minimal volume, and let $Q_{r} \in \mathcal{F}_{r}$ have minimal surface area.

The minima do exist according to the Blaschke Selection Theorem, and all extreme points of $P_{r}$ and $Q_{r}$ lie in $r S^{2}$ by the monotonicity of the volume and surface area. Our main result is

Theorem 2.1. If $r=\sqrt{3}$ or $r=\sqrt{15-6 \sqrt{5}}$ then any $P_{r}$ is an octahedron, or an icosahedron, respectively, circumscribed around $B^{3}$. Moreover the analogous statements hold for $Q_{r}$.

Remark. In the high-dimensional case no analogue of Theorem 2.1 can be expected. More precisely cylinders whose bases are unit $(n-1)$-balls show that $n$-simplices and $n$ dimensional cubes are not optimal in their class if $n \geq 4$, and the regular $n$-crosspolytope is not optimal in its class if $n \geq 8$.

Returning to the three-dimensional case, cylinders whose bases are unit discs show that the analogues of Theorem 2.1 do not hold for the cube and for the dodecahedron. However, we suspect that the regular tetrahedron is optimal in its class:

Conjecture 2.2. If $r=3$ then all $P_{r}$ and $Q_{r}$ are regular tetrahedra.

Concerning Conjecture 2.2, the proof of Theorem 2.1 breaks down because "regular triangles are no longer optimal faces"; namely, Lemma 4.1 does not hold for $r=3$ (see Remark 4.2). It is a challenging problem to find the analogue of Theorem 2.1 for the mean width in place of the volume and the surface area.

In subsequent papers, Böröczky and Böröczky [2] provide a stability version of Theorem 2.1, and Böröczky et al. [3] describe asymptotic properties of $P_{r}$ or $Q_{r}$ when $r$ is close to 1. This last problem; namely, the case if $r$ is close to 1, is meaningful also in higher dimensions (see [4]).

The paper is structured in the following way: Section 3 recalls some known facts about orthoschemes. Section 4 proves Lemma 4.1, which forms the core of the whole argument, and states that optimal faces are regular triangles. Finally Theorem 2.1 is proved in Section 5.

\section{Orthoschemes and the Density of the Surface Area}

We observe that if $M \in \mathcal{F}_{r}$ then $S\left(B^{3}\right) / S(M)$ is maximal for $M=Q_{r}$. This approach suggests the following definition: We write $\pi(\cdot)$ to denote the radial projection onto $S^{2}$. 
If $F$ is a convex domain in $\mathbb{E}^{3}$ whose affine hull avoids int $B^{3}$ then let

$$
d(F)=\frac{A(\pi(F))}{A(F)} .
$$

It aff $F$ touches $B^{3}$ then $d(F)$ is the density of the part of $B^{3}$ contained in $\operatorname{conv}\{o, F\}$ with respect to $\operatorname{conv}\{o, F\}$.

Next we say that a tetrahedron $S=\operatorname{conv}\left\{o, v_{1}, v_{2}, v_{3}\right\}$ is an orthoscheme if $v_{1}$ is orthogonal to aff $\left\{v_{1}, v_{2}, v_{3}\right\}$, and $v_{3}-v_{2}$ is orthogonal to aff $\left\{o, v_{1}, v_{2}\right\}$. Naturally the order of vertices is important. If $\varrho_{i}=\left\|v_{i}\right\|$ for $i=1,2,3$ then we call $S$ a $\left(\varrho_{1}, \varrho_{2}, \varrho_{3}\right)$ orthoscheme. Bezdek [1] proved the following result:

Lemma 3.1 (Bezdek). For $i=1,2$, let $S_{i}=\operatorname{conv}\left\{o, v_{i 1}, v_{i 2}, v_{i 3}\right\}$ be an orthoscheme in $\mathbb{E}^{3}$, and let $R_{i}=\operatorname{conv}\left\{v_{i 1}, v_{i 2}, v_{i 3}\right\}$. If $1 \leq\left\|v_{1 j}\right\| \leq\left\|v_{2 j}\right\|$ for $j=1,2,3$ then

$$
d\left(R_{1}\right) \geq d\left(R_{2}\right),
$$

with equality if and only if $S_{1}$ and $S_{2}$ are congruent.

\section{The Optimal Faces Are Regular Triangles}

We prove Theorem 2.1 by showing that the optimal faces are regular triangles.

Lemma 4.1. Given $r \in(1, \sqrt{3}]$, let $D$ be a circular disc of radius $\sqrt{r^{2}-1}$ that touches $B^{3}$ in the centre of $D$. If $T$ is any triangle whose vertices lie on $\partial D$ then

$$
d(T) \leq \frac{8 \arctan (\sqrt{3}(r-1) /(3+r))}{\sqrt{3}\left(r^{2}-1\right)},
$$

with equality if and only if $T$ is a regular triangle.

Remark 4.2. We note that the regular tetrahedron circumscribed around the unit ball is of circumradius 3. Now if $T$ is a narrow isosceles triangle then $d(T)$ is close to $1 / r$ (see Proposition 4.5(ii)), which is larger than $8 \arctan (\sqrt{3}(r-1) /(3+r)) / \sqrt{3}\left(r^{2}-1\right)$ if $r \geq 3$.

The whole section is dedicated to proving Lemma 4.1, and to verifying two corollaries. First we introduce some notation, and the notion of density of variation. Then we verify the auxiliary statements of Propositions 4.3-4.7, which will be used in the proof of Lemma 4.1.

During the proof of Lemma 4.1, $T$ always denotes some triangle whose vertices lie in $\partial D$. Moreover the sides of $T$ and their lengths are denoted by $a, b$ and $c$, and the distances of the midpoints of $a, b$ and $c$ from $o$ are denoted by $m_{a}, m_{b}$ and $m_{c}$, respectively. We plan to compare $T$ with the regular triangle $T_{*}$ inscribed into $D$. We write $a_{*}$ to denote the common length of the sides of $T_{*}$, and $m_{*}$ to denote the common distance of the midpoints of the sides of $T_{*}$ from $o$. 
In the course of the argument, we consider $T$ as part of certain family $T(s)$ where the vertices of $T(s)$ are differentiable functions of the real parameter $s$. Our main approach is to investigate the density of the variation; namely,

$$
v(T(s))=\frac{(\partial / \partial s) A(\pi(T(s)))}{(\partial / \partial s) A(T(s))} .
$$

Reparametrization does not change the density of variation. When it is clear from the context what the family $T(s)$ is then we drop the reference to $s$. Let us explain the role of $v(T(s))$ : If $A(T(s))$ is a strictly monotone function of $s$ on an interval $\left[s_{1}, s_{2}\right]$ then the Cauchy Mean Value theorem provides $s \in\left(s_{1}, s_{2}\right)$ satisfying

$$
\begin{aligned}
d\left(T\left(s_{2}\right)\right)-d\left(T\left(s_{1}\right)\right) & =\left[v(T(s))-d\left(T\left(s_{2}\right)\right)\right] \cdot \frac{A\left(T\left(s_{2}\right)\right)-A\left(T\left(s_{1}\right)\right)}{A\left(T\left(s_{1}\right)\right)} \\
& =\left[v(T(s))-d\left(T\left(s_{1}\right)\right)\right] \cdot \frac{A\left(T\left(s_{2}\right)\right)-A\left(T\left(s_{1}\right)\right)}{A\left(T\left(s_{2}\right)\right)} .
\end{aligned}
$$

One of the reasons why the notion of density of variation is so useful is that it satisfies the simple formulae in Propositions 4.3 and 4.4.

Proposition 4.3. If $a<b$, and $T$ is deformed in a way that the side $c$ is kept fixed and $a$ is increased then $A(T)$ is strictly increasing, and

$$
v(T)=\frac{r}{m_{a}^{2} m_{b}^{2}} .
$$

Proof. The statement about $A(T)$ readily holds. Turning to the formula for $v(T)$, we write the same symbol to denote a Euclidean segment and the length of it. Moreover, we write $w_{a}$ and $w_{b}$ to denote the common endpoint of $a$ and $c$, and of $b$ and $c$, respectively.

Let $\widetilde{T}$ be the new position of $T$. Then $\widetilde{T}$ is obtained from $T$ by attaching a triangle $T_{b}$ and removing a triangle $T_{a}$. We observe that $w_{a}$ and $w_{b}$ are vertices of $T_{a}$ and $T_{b}$, respectively, and write $\delta$ to denote the common measure (in radians) of the angles of $T_{a}$ and $T_{b}$ at $w_{a}$ and $w_{b}$, respectively. In particular,

$$
A\left(T_{a}\right) \sim \frac{1}{2} a^{2} \cdot \delta \quad \text { and } \quad A\left(T_{b}\right) \sim \frac{1}{2} b^{2} \cdot \delta
$$

where $\sim$ means that the ratio of the two sides tends to one as $\delta$ tends to zero. Therefore

$$
A(\widetilde{T})-A(T) \sim \frac{1}{2}\left(b^{2}-a^{2}\right) \cdot \delta=2\left(m_{a}^{2}-m_{b}^{2}\right) \cdot \delta,
$$

where we used that $m_{a}^{2}+(a / 2)^{2}=r^{2}=m_{b}^{2}+(b / 2)^{2}$.

It can be deduced say by repeated application of the cross-product that the angle of $\pi\left(T_{a}\right)$ at $\pi\left(w_{a}\right)$ is asymptotically $(r \cdot \delta) / m_{a}^{2}$ as $\delta$ tends to zero, and it is easy to see that the two corresponding spherical sides of $\pi\left(T_{a}\right)$ are both asymptotically $2 \arcsin (a / 2 r)$. We compare the area of $T_{a}$ with the area of the spherical circular sector $S_{a}$ of radius $\varrho=2 \arcsin (a / 2 r)$ and angle $(r \cdot \delta) / m_{a}^{2}$. Since

$$
A\left(S_{a}\right)=\frac{r \delta}{m_{a}^{2}}(1-\cos \varrho)=\frac{2 r \delta}{m_{a}^{2}} \sin ^{2} \frac{\varrho}{2}=\frac{2 r \delta}{m_{a}^{2}}\left(\frac{a}{2 r}\right)^{2}=\left(\frac{2 r}{m_{a}^{2}}-\frac{2}{r}\right) \cdot \delta,
$$


we deduce that

$$
A\left(\pi\left(T_{a}\right)\right) \sim A\left(S_{a}\right)=\left(\frac{2 r}{m_{a}^{2}}-\frac{2}{r}\right) \cdot \delta .
$$

Using the analogous formula for $A\left(\pi\left(T_{b}\right)\right)$ leads to

$$
A(\pi(\widetilde{T}))-A(\pi(T)) \sim \frac{2 r}{m_{a}^{2} m_{b}^{2}} \cdot\left(m_{a}^{2}-m_{b}^{2}\right) \cdot \delta .
$$

Therefore dividing (4) by (3) proves Proposition 4.3.

Proposition 4.4. Let $b=c$, and let $T$ be deformed in a way that $T$ stays isosceles, and side $a$ is increased. If $a<a_{*}$ then $A(T)$ is strictly increasing, and if $a>a_{*}$ then $A(T)$ is strictly decreasing. Moreover,

$$
v(T)=\frac{r}{m_{a}^{2} m_{b}^{2}} .
$$

Proof. We may parametrize $T$ by $a$ as the family $T(a)$. Let us first slightly move only one endpoint of $a$ (hence $T$ becomes a non-isosceles triangle), and afterwards move the other endpoint of $a$ in a way that $T$ again becomes isosceles. Now we conclude Proposition 4.4 by Proposition 4.3.

Let us determine some specific values of the density and the density of variation.

Proposition 4.5. Assuming that $b=c$ and $T$ has no obtuse angle, let us parametrize $T$ as $T(a)$ where $0<a \leq 2 \sqrt{r^{2}-1}$. Then

(i) we have

$$
d\left(T\left(a_{*}\right)\right)=d\left(T_{*}\right)=\frac{8 \arctan (\sqrt{3}(r-1) /(3+r))}{\sqrt{3}\left(r^{2}-1\right)} \quad \text { at } \quad a=a_{*} ;
$$

(ii) and $\lim _{a \rightarrow 0} d(T(a))=\lim _{a \rightarrow 0} v(T(a))=1 / r$ as a tends to zero;

(iii) moreover, $v\left(T\left(2 \sqrt{r^{2}-1}\right)\right)=2 r /\left(1+r^{2}\right)$ at $a=2 \sqrt{r^{2}-1}$.

Proof. The statements about $v(T(a))$ readily follow from Proposition 4.4. Concerning density, let $q$ be the centre of $D$. Now if $R$ is a triangle with a right angle such that the longest side connects $q$ to a point of $\partial D$, and the angle of $R$ at $q$ is $\omega$ then

$$
A(R)=\frac{\left(r^{2}-1\right) \tan \omega}{2\left(1+\tan ^{2} \omega\right)} \quad \text { and } \quad A(\pi(R))=\arctan \frac{(r-1) \tan \omega}{r+\tan ^{2} \omega} .
$$

If $a<2 \sqrt{r^{2}-1}$ and the angle of $T(a)$ opposite to $a$ is $\alpha$ then $T(a)$ can be dissected into six such triangles where $\omega=\alpha$ for two of the triangles, and $\omega=(\pi-\alpha) / 2$ for the other four triangles. In turn simple calculations yield Proposition 4.5.

Next we compare the quantities occurring in Proposition 4.5. 
Proposition 4.6. We have the inequalities $1 / r<d\left(T_{*}\right)<r / m_{*}^{4}<2 r /\left(1+r^{2}\right)$.

Proof. The inequality $r / m_{*}^{4}<2 r /\left(1+r^{2}\right)$ follows by $m_{*}=\sqrt{r^{2}+3} / 3$. Since $\arctan t<t$ for positive $t$, we deduce by $1<r \leq \sqrt{3}$ that

$$
\begin{aligned}
\frac{r}{m_{*}^{4}}-d\left(T_{*}\right) & >\frac{16 r}{\left(3+r^{2}\right)^{2}}-\frac{8}{(3+r)(1+r)} \\
& =\frac{8\left(-r^{3}+r^{2}+3 r+9\right) \cdot(r-1)}{\left(3+r^{2}\right)^{2}(3+r)(1+r)}>0 .
\end{aligned}
$$

Finally we observe that $\arctan t>t-\frac{1}{3} t^{3}$, and obtain by $1<r \leq \sqrt{3}$ that

$$
\begin{aligned}
d\left(T_{*}\right)-\frac{1}{r} & >\frac{8}{\sqrt{3}\left(r^{2}-1\right)} \cdot\left[\frac{\sqrt{3}(r-1)}{3+r}-\frac{1}{3}\left(\frac{\sqrt{3}(r-1)}{3+r}\right)^{3}\right]-\frac{1}{r} \\
& =\frac{\left(27-10 r-r^{2}\right) \cdot(r-1)}{r(r+3)^{3}}>0 .
\end{aligned}
$$

Proposition 4.7. There exists positive $a_{0}<a_{*}$ with the following property: Assuming that $b=c$ and $T$ has no obtuse angle,

$$
\begin{aligned}
& \text { if } a<a_{0} \quad \text { then } \frac{r}{m_{a}^{2} m_{b}^{2}}<d\left(T_{*}\right) \text {; } \\
& \text { if } a>a_{0} \quad \text { then } \frac{r}{m_{a}^{2} m_{b}^{2}}>d\left(T_{*}\right) .
\end{aligned}
$$

Proof. Let $2 \omega$ be the angle of $T$ opposite to $a$, and let $s=\sin ^{2} \omega$. In particular $s \in\left(0, \frac{1}{2}\right]$ where $s=\frac{1}{2}$ and $s=\frac{1}{4}$ correspond to the cases when $T$ has a right angle and is regular, respectively, and if $s$ tends to zero then $T$ approaches a diameter of $D$. Writing $\Omega=r^{2}-1$, we have

$$
m_{a}^{2} m_{b}^{2}=f(s) \quad \text { for } \quad f(s)=\left[1+\Omega(1-2 s)^{2}\right] \cdot(1+\Omega s) .
$$

We deduce by Proposition 4.6 that

$$
\frac{r}{f(0)}<d\left(T_{*}\right) \quad \text { and } \quad \frac{r}{f\left(\frac{1}{2}\right)}>\frac{r}{f\left(\frac{1}{4}\right)}>d\left(T_{*}\right) .
$$

We observe that

$$
f^{\prime}(s)=12 \Omega^{2} s^{2}+8\left(\Omega-\Omega^{2}\right) s+\Omega^{2}-3 \Omega,
$$

hence $0<\Omega \leq 2$ implies that $f^{\prime}(0)<0$ and $f^{\prime}\left(\frac{1}{2}\right)=\Omega>0$. Since $f^{\prime}$ is quadratic in $s$ with a positive main coefficient, we deduce that $f$ is first decreasing then increasing on $\left[0, \frac{1}{2}\right]$, hence (6) yields Proposition 4.7. 
Proof of Lemma 4.1. We consider several cases. In each case we prove that $d(T)<$ $d\left(T_{*}\right)$ holds provided that $T$ is not congruent to $T_{*}$.

Case 1: The angle of $T$ opposite to $a$ is obtuse, and $c \leq b \leq a_{*}$. We write $q$ to denote the centre of $D$. Moreover, let $T_{a}, T_{b}$ and $T_{c}$ denote the convex hulls of $q$ on the one hand, and $a, b$ and $c$, respectively, on the other hand. We observe that $\operatorname{conv}\left\{o, T_{*}\right\}$ can be dissected into six $\left(1, m_{*}, r\right)$-orthoschemes. In addition, $\operatorname{conv}\left\{o, T_{a}\right\}, \operatorname{conv}\left\{o, T_{b}\right\}$ and $\operatorname{conv}\left\{o, T_{c}\right\}$ can be dissected into two $\left(1, m_{a}, r\right)$-orthoschemes, $\left(1, m_{b}, r\right)$-orthoschemes and $\left(1, m_{c}, r\right)$-orthoschemes, respectively. Now Lemma 3.1 and $m_{b}, m_{c} \geq m_{*}$ yield that $d\left(T_{b}\right), d\left(T_{c}\right) \leq d\left(T_{*}\right)$. Since $m_{a}<m_{b}, m_{c}$, it follows by Lemma 3.1 that $d\left(T_{a}\right)>$ $d\left(T_{b}\right), d\left(T_{c}\right)$. Now $T$ is the difference of $T_{b} \cup T_{c}$ and $T_{a}$, thus

$$
d(T)<\max \left\{d\left(T_{b}\right), d\left(T_{c}\right)\right\} \leq d\left(T_{*}\right) .
$$

Case 2: $b=c$, and the angle of $T$ opposite to a is at most $\pi / 2$. We may parametrize $T$ by $a$ as the family $T(a), 0<a \leq 2 \sqrt{r^{2}-1}$. If $a>a_{*}$ then we use that $A(T(a))$ is strictly decreasing, moreover, $d\left(T\left(a_{*}\right)\right)=d\left(T_{*}\right)$ and $v(T(a))>d\left(T_{*}\right)$ for $a>a_{*}$ according to Propositions 4.4 and 4.7. Applying (2) to $\left[a_{*}, a\right]$ yields that $d(T(a))<d\left(T_{*}\right)$ when $a>a_{*}$.

If $a_{0} \leq a \leq a_{*}$ then we use that $d\left(T\left(a_{*}\right)\right)=d\left(T_{*}\right)$ and $v(T(a))>d\left(T_{*}\right)$ for $a \in\left(a_{0}, a_{*}\right)$ according to Propositions 4.4 and 4.7. Applying (1) to $\left[a, a_{*}\right]$ shows that $d(T(a))<d\left(T_{*}\right)$ when $a_{0} \leq a<a_{*}$.

Finally, assume that $a<a_{0}$. Since $\lim _{s \rightarrow 0} d(T(s))=1 / r$ (see Proposition 4.5(ii)) and $d(T(s))$ is continuous, Proposition 4.6 provides $a_{1} \in(0, a)$ satisfying $d\left(T\left(a_{1}\right)\right)<d\left(T_{*}\right)$. Therefore applying (1) to $\left[a_{1}, a\right]$ yields the existence of some $s \in\left(a_{1}, a\right)$ satisfying

$$
d\left(T(a)=\frac{A\left(T\left(a_{1}\right)\right)}{A(T(a))} \cdot d\left(T\left(a_{1}\right)\right)+\left(1-\frac{A\left(T\left(a_{1}\right)\right)}{A(T(a))}\right) \cdot v(T(s)) .\right.
$$

Since $v(T(s))<d\left(T_{*}\right)$ according to Proposition 4.7, we deduce $d(T(a))<d\left(T_{*}\right)$.

In summary $d(T)<d\left(T_{*}\right)$ holds in Case 2 provided that $a \neq a_{*}$.

Case 3: $b>a \geq a_{*}$. We fix $c$, and deform $T=T(a)$ in a way that $a$ increases until $T$ becomes isosceles at $a=a_{2}$. In particular, $A(T(a))$ strictly increases. Now $m_{b}<m_{a} \leq m_{*}$ holds at any position, hence Proposition 4.6 yields that

$$
v(T(a))>\frac{r}{m_{*}^{4}}>d\left(T_{*}\right)
$$

for any $a$. Since $d\left(T\left(a_{2}\right)\right)<d\left(T_{*}\right)$ (see Case 2), we deduce by (1) that $d(T)<d\left(T_{*}\right)$.

Case 4: $c<b \leq a_{*}$. If the angle of $T$ opposite to $a$ is obtuse then $d(T)<d\left(T_{*}\right)$ according to Case 1. Therefore we assume that the angle of $T$ opposite to $a$ is at most $\pi / 2$. In this case $a \geq a_{*}$, and we claim that

$$
\frac{r}{m_{a}^{2} m_{b}^{2}}>d\left(T_{*}\right)
$$

If $a$ is fixed and $c \leq b \leq a_{*}$ then $m_{b}$ attains its maximum exactly when $b=c$. Therefore Proposition 4.7 yields (9). 
Now we fix $c$, and deform $T=T(b)$ in a way that $b$ increases until $b=a$ at some $b=b_{2}$, hence $A(T(b))$ strictly increases. Since $v(T(b))>d\left(T_{*}\right)$ for any $b$ according to (9), and $d\left(T\left(b_{2}\right)\right)<d\left(T^{*}\right)$ according to Case 2 , we conclude by (1) that $d(T)<d\left(T_{*}\right)$. Therefore $d(T)<d\left(T_{*}\right)$ holds in Case 4.

Since $T$ has two sides whose lengths are either both at least $a_{*}$ or both at most $a_{*}$, any $T$ belongs to at least one of Cases $1-4$ above. Therefore the proof of Lemma 4.1 is now complete.

The following consequence of Lemmas 3.1 and 4.1 will be actually used in the proof of Theorem 2.1:

Corollary 4.8. Let $r \in(1, \sqrt{3}]$. If $F$ is a polygon whose vertices lie on $r S^{2}$ and aff $F$ avoids the interior of $B^{3}$ then

$$
d(F) \leq \frac{8 \arctan (\sqrt{3}(r-1) /(3+r))}{\sqrt{3}\left(r^{2}-1\right)},
$$

with equality if and only if $F$ is a regular triangle of circumradius $\sqrt{r^{2}-1}$.

Proof. Triangulating $F$ by diagonals starting from a fixed vertex of $F$ shows that we may assume that $F$ is a triangle $T$.

Writing $D=$ aff $F \cap r B^{3}$, let $\varrho$ be the distance of aff $D$ from $o$, and let $\widetilde{T}$ be a regular triangle inscribed into $D$. In addition let $T_{*}$ be some regular triangle whose affine hull touches $B^{3}$, and whose vertices lie on $r S^{2}$, hence $d\left(T_{*}\right)=8 \arctan (\sqrt{3}(r-1) /(3+r)) /$ $\sqrt{3}\left(r^{2}-1\right)$. Now the vertices of both $(1 / \varrho) T$ and $(1 / \varrho) \widetilde{T}$ lie on $(r / \varrho) S^{2}$, therefore Lemma 4.1 yields that $d(T) \leq d(\widetilde{T})$. Since $d(\widetilde{T}) \leq d\left(T_{*}\right)$ according to Lemma 3.1, we conclude Corollary 4.8 .

In order to handle the equality case of Theorem 2.1 , we need a weak stability version of Corollary 4.8. We say that the compact convex sets $M$ and $N$ are $\varepsilon$-close for $\varepsilon>0$ if there exist congruent copies $M^{\prime}$ and $N^{\prime}$ of $M$ and $N$, respectively, satisfying

$$
\frac{1}{1+\varepsilon} N^{\prime} \subset M^{\prime} \subset(1+\varepsilon) N^{\prime}
$$

Naturally in this case the dimensions of $M$ and $N$ coincide.

Corollary 4.9. Let $r=\sqrt{3}$ or $r=\sqrt{15-6 \sqrt{5}}$. For any $\varepsilon \in(0,1)$ there exists $\mu>0$ with the following property: If $F$ is a polygon whose vertices lie on $r S^{2}$, aff $F$ avoids the interior of $B^{3}$, and

$$
d(F) \geq(1+\mu)^{-1} \frac{8 \arctan (\sqrt{3}(r-1) /(3+r))}{\sqrt{3}\left(r^{2}-1\right)},
$$

then $F$ is $\varepsilon$-close to a regular triangle of circumradius $\sqrt{r^{2}-1}$. 
Proof. Again we may assume that $F$ is a triangle $T$. Let $T^{\prime}$ be a regular triangle in aff $T$ whose vertices lie on $r S^{2}$, and let $T_{*}$ be a regular triangle that touches $B^{3}$, and whose vertices lie on $r S^{2}$. In particular,

$$
d\left(T_{*}\right)=\frac{8 \arctan (\sqrt{3}(r-1) /(3+r))}{\sqrt{3}\left(r^{2}-1\right)} .
$$

Let us assume that (10) holds for a suitable positive $\mu$. Since $d(T) \leq d\left(T^{\prime}\right) \leq d\left(T_{*}\right)$ according to Lemmas 4.1 and 3.1, the equality case of Lemma 3.1 shows that if $\mu$ is small enough then $T^{\prime}$ is $(\varepsilon / 3)$-close to $T_{*}$. The proof of Lemma 4.1 shows first that $T$ cannot be close to a segment, and secondly that if $\mu$ is small enough then $T$ has to be $(\varepsilon / 3)$-close to $T^{\prime}$. In turn we conclude Corollary 4.9.

\section{Proof of Theorem 2.1}

Let $r=\sqrt{3}$ or $r=\sqrt{15-6 \sqrt{5}}$, and let $C \in \mathcal{F}_{r}$. It is sufficient to consider the case of surface area because $V(C) \geq 3 S(C)$, and $V(C)=3 S(C)$ if $C$ is the octahedron when $r=\sqrt{3}$, or the icosahedron when $r=\sqrt{15-6 \sqrt{5}}$. We may assume that the extreme points of $C$ lie on $r S^{2}$. We define $T_{*}$ to be a regular triangle that touches $B^{3}$, and whose vertices lie on $r S^{2}$.

Let us construct a sequence of polytopal elements of $\mathcal{F}_{r}$ tending to $C$. For any integer $n \geq 1$, let $\Sigma_{n}$ be a finite $(1 / n)$-net in $r S^{2}$; namely, $\Sigma_{n} \subset r S^{2}$ and $r S^{2} \subset \Sigma_{n}+(1 / n) B^{3}$. We define $M_{n}$ to be the convex hull of all points $x$ of $\Sigma_{n}$ such that $\|x-y\| \leq 2 / n$ for some $y \in \operatorname{ext} C$. Then $M_{n}$ tends to $C$, and it is easy to see that $M_{n} \in \mathcal{F}_{r}$.

Applying Corollary 4.8 to each face of $M_{n}$ yields that

$$
S\left(M_{n}\right) \geq \frac{4 \pi}{d\left(T_{*}\right)},
$$

hence (11) holds for $C$ in place of $M_{n}$. Since we have equality in (11) if all faces are regular triangles touching $B^{3}$, we conclude Theorem 2.1 without the case of equality.

In order to characterize the equality case in Theorem 2.1, we assume that $C$ is not a regular octahedron if $r=\sqrt{3}$, and not a regular icosahedron if $r=\sqrt{15-6 \sqrt{5}}$. We call the intersection of $C$ with a supporting plane a generalized face of $C$ (that might be a single point). Now there exist positive $\varepsilon_{0}$ and $v_{0}$ with the following properties: Writing $\Psi_{0}$ to denote the union of generalized faces of $C$ that are $\varepsilon_{0}$-close to $T_{*}$, and setting $\Omega_{0}=\partial C \Psi_{0}$, we have $A\left(\pi\left(\Omega_{0}\right)\right)>2 v_{0}$. We may also assume that if a polygon $F$ is $\varepsilon_{0}$-close to $T_{*}$, and $x$ is the circumcentre of $F$ then $F$ contains a circular disc centred at $x$ of radius 0.1 .

For any $M_{n}$, we write $\Psi_{n}$ to denote the union of faces of $M_{n}$ that are $\varepsilon_{0}$-close to $T_{*}$, and set $\Omega_{n}=\partial M_{n} \backslash \Psi_{n}$. We observe that if $\left\{M_{n^{\prime}}\right\}$ is a subsequence of $\left\{M_{n}\right\}$, and $F_{n^{\prime}}$ is a face of $M_{n^{\prime}}$ that is $\varepsilon_{0}$-close to $T_{*}$, and $F_{n^{\prime}}$ tends to a compact convex set $F$ then $F$ is a generalized face of $C$ that is $\varepsilon_{0}$-close to $T_{*}$. Therefore we have $A\left(\pi\left(\Omega_{n}\right)\right)>v_{0}$ for large $n$. Let $\mu_{0}$ be the value of $\mu$ provided by Corollary 4.9 for $\varepsilon=\varepsilon_{0}$. We deduce for large $n$ that

$$
S\left(M_{n}\right) \geq \frac{4 \pi-v_{0}}{d\left(T_{*}\right)}+\left(1+\mu_{0}\right) \frac{v_{0}}{d\left(T_{*}\right)} \geq\left(1+\frac{\mu_{0} \nu_{0}}{4 \pi}\right) \frac{4 \pi}{d\left(T_{*}\right)} .
$$


Since (12) holds also for $C$ in place of $M_{n}$, it follows that $S(C)>S\left(Q_{r}\right)$, which in turn completes the proof of Theorem 2.1.

\section{Acknowledgements}

We thank Peter M. Gruber for helpful discussions, and the referees for helping the paper to get focused.

\section{References}

1. K. Bezdek: On a stronger form of Roger's lemma and the minimum surface area of Voronoi cells in unit ball packings. J. Reine Angew. Math., 518 (2000), 131-142.

2. K. Böröczky, K.J. Böröczky: A stability property of the octahedron and the icosahedron. Submitted. Available at www.renyi.hu/ carlos/radiusstab.pdf.

3. K. Böröczky, K.J. Böröczky, G. Wintsche: Typical faces of extremal polytopes with respect to a thin threedimensional shell. Period. Math Hungar., accepted. Available at www.renyi.hu/ carlos/radiustyp.pdf.

4. K. Böröczky, K.J. Böröczky, C. Schütt, G. Wintsche: Convex bodies of minimal volume, surface area and mean width with respect to thin shells. Canad. J. Math., accepted. Available at www.renyi.hu/ carlos/radiusasymp.pdf.

5. K. Böröczky, K. Máthéné Bognár: Regular polyhedra and Hajós polyhedra. Studia Sci. Math. Hungar., 35 (1999), 415-426.

6. L. Fejes Tóth: Regular Figures. Pergamon Press, Oxford, 1964.

7. J. Molnár: Alcune generalizzazioni del teorema di Segre-Mahler. Atti Accad. Naz. Lincei Rend. Cl. Sci. Fis. Mat. Natur. (8), 30 (1961), 700-705.

8. R. Schneider. Convex Bodies-the Brunn-Minkowski Theory. Cambridge University Press, Cambridge, 1993.

Received March 7, 2005, and in revised form September 27, 2005, and November 2, 2006.

Online publication July 13, 2007. 